\title{
Criterion-related validity of the last 7-day, short form of the International Physical Activity Questionnaire in Swedish adults
}

\author{
Ulf Ekelund ${ }^{1,2, *}$, Hanna Sepp ${ }^{3}$, Sören Brage ${ }^{1}$, Wulf Becker $^{3}$, Rupert Jakes ${ }^{1}$, \\ Mark Hennings ${ }^{4}$ and Nicholas J Wareham ${ }^{1}$ \\ 'Medical Research Council Epidemiology Unit, Elsie Widdowson Laboratory, Fulbourn Road, Cambridge CB1 9NL, \\ UK: ${ }^{2}$ Department of Physical Education and Health, Örebro University, Örebro, Sweden: ${ }^{3}$ National Food \\ Administration, Uppsala, Sweden: ${ }^{4}$ Sidney Sussex College, Cambridge, UK
}

Submitted 27 January 2005: Accepted 15 June 2005

\begin{abstract}
Objective: To examine the validity of the short, last 7-day, self-administered form of the International Physical Activity Questionnaire (IPAQ).

Design: All subjects wore an accelerometer for seven consecutive days and completed the IPAQ questionnaire on the eighth day. Criterion validity was assessed by linear regression analysis and by modified Bland-Altman analysis. Specificity and sensitivity were calculated for classifying respondents according to the physical activity guidelines of the American College of Sports Medicine/Centers for Disease Control and Prevention.

Setting: Workplaces in Uppsala, Sweden.

Subjects: One hundred and eighty-five (87 males) participants, aged 20 to 69 years. Results: Total self-reported physical activity (PA) (MET-min day ${ }^{-1}$ ) was significantly correlated with average intensity of activity (counts $\mathrm{min}^{-1}$ ) from accelerometry $(r=0.34, P<0.001)$. Gender, age, education and body mass index did not affect this relationship. Further, subcomponents of self-reported PA (time spent sitting, time in PA, time in moderate and vigorous activity (MVPA)) were significantly correlated with objectively measured PA $(P<0.05)$. Self-reported time in PA was significantly different from time measured by accelerometry (mean difference: -25.9 min day $^{-1}$; $95 \%$ limits of agreement: -172 to 120 min day $\left.^{-1} ; P<0.001\right)$. IPAQ identified $77 \%$ (specificity) of those who met the current PA guidelines of accumulating more than 30 min day $^{-1}$ in MVPA as determined by accelerometry, whereas only $45 \%$ (sensitivity) of those not meeting the guidelines were classified correctly.

Conclusions: Our results indicate that the short, last 7-days version of the IPAQ has acceptable criterion validity for use in Swedish adults. However, the IPAQ instrument significantly overestimated self-reported time spent in PA. The specificity to correctly classify people achieving current PA guidelines was acceptable, whereas the sensitivity was low.
\end{abstract}

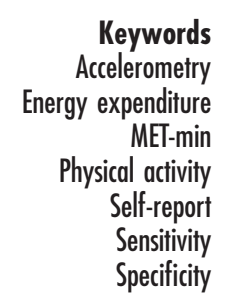

Physical activity (PA) has been defined as any bodily movement produced by skeletal muscles which results in energy expenditure above the basal level ${ }^{1}$. Measurement of PA, and energy expenditure associated with it, comprises time (duration), number of sessions (frequency) and intensity ${ }^{2}$. Based on these variables, selfreported energy expenditure (EE) attributable to PA may be estimated ${ }^{2}$.

However, the measurement of PA in large-scale epidemiological studies and surveillance systems is difficult due to its complex nature ${ }^{3}$. Until recently, no standardised self-report instrument has been available for cross-cultural comparisons.

The International Physical Activity Questionnaire (IPAQ) was developed in an attempt to standardise assessment of the prevalence of PA in different countries and cultures around the world ${ }^{4}$. Eight different forms of IPAQ were developed, and the reliability and validity of these forms were tested in 14 centres in 12 countries during the year 2000. From this study, it was suggested that the last 7-day, short form of the IPAQ could be used for national and regional prevalence studies ${ }^{4}$. Despite globally acceptable measurement properties, the results from some study locations indicated limited validity of the last 7-day, short-form IPAQ ${ }^{4}$. Moreover, the absolute validity (i.e. the validity to assess the absolute levels of activity) of IPAQ was not addressed.

The purpose of the present study was therefore to assess the criterion and absolute validity of the last 7-day, selfadministered, short form of the IPAQ in a random sample of adult males and females from Sweden, using accelerometry as the criterion instrument. As a secondary 
aim, we compared objectively measured PA with the response to two previous questions used in Swedish health survey systems.

\section{Methods}

\section{Subjects and design}

Subjects were selected randomly from different workplaces, one student union and by advertisement in a daily newspaper in Uppsala, Sweden. Workplaces were selected with the aim of increasing variability in age, gender and education level of the potential respondents. A total of 198 subjects ( 93 males) aged 20 to 69 years agreed to participate in the study. Demographic characteristics, including education level, were obtained by questionnaire on day 1 of the study. Height and weight were measured by standard clinical techniques. Body mass index (BMI) was calculated as body weight divided by the square of height $\left(\mathrm{kg} \mathrm{m}^{-2}\right)$.

The volunteers were thereafter instructed on how to wear the activity monitor (MTI Actigraph), which was initialised to record physical activity from day 2 and onwards. Subjects were contacted again on day 8, when the activity monitor was collected and the PA questionnaire administered. The ethics committee of Örebro University, Sweden, approved the research protocol and all participants provided written informed consent.

\section{PA by self-report}

Self-reported PA was obtained by the last 7 days, short, self-administered version of the IPAQ. No official Swedish version of IPAQ was available (www.ipaq.ki.se). However, the version used in this study was the same as in the 12 -country reliability and validity study ${ }^{4}$. The questionnaire collects information on time (i.e. number of sessions and average time per session) spent walking, in moderateintensity PA, in vigorous-intensity PA and sitting, on weekdays and weekend days. Questions regarding participation in moderate and vigorous PA were supplemented by concrete examples of activities commonly performed. Data from the questionnaire were summed within each item (i.e. vigorous intensity, moderate intensity, walking) to estimate the total amount of time spent in PA per week. Total daily PA (MET-min day ${ }^{-1}$ ) was estimated by summing the product of reported time within each item by a MET value specific to each category of PA and expressed as a daily average MET score (where MET is metabolic equivalent; 1 MET = resting energy expenditure) according to the official IPAQ scoring protocol (www.ipaq.ki.se). Vigorous intensity of PA was assumed to correspond to $8 \mathrm{METs}$, moderate-intensity activity to 4 METs and walking to 3.3 METs (www.ipaq.ki.se). Data were thereafter cleaned for out-of-range values (i.e. total MET-min week ${ }^{-1}$ greater than three standard deviations ( $>3 \mathrm{SD}$ ) from the mean; $n=12$ ). Based on the selfreported time spent walking and in moderate- and vigorous-intensity physical activity (MVPA), subjects were categorised as being sufficiently and insufficiently active according to the American College of Sports Medicine (ACSM)/Centers for Disease Control and Prevention (CDC) guidelines 5 , i.e. accumulating at least $30 \mathrm{~min}$ of moderate-intensity activity per day.

In addition to the IPAQ questions, we also included two categorical questions about PA at work and during leisure time. Statistics Sweden (SCB), in their annual surveys examining the living conditions in the adult (16-65 years) Swedish population (Undersökningar om levnadsförhållanden, ULF; www.scb.se), has previously used a similar question about leisure-time PA. The validity of this question has not been formally tested. The categorical question regarding leisure-time PA referred to activity during the last 12 months and the respondents were asked to average their amount of leisure-time PA in four categories: (1) sedentary leisure-time (i.e. $<2 \mathrm{~h}$ of activity such as walking and biking per week); (2) sporadic leisure-time moderate activity (i.e. at least $2 \mathrm{~h}$ of moderate-intensity activity such as bicycling, walking and gardening per week); (3) sporadic regular exercise (i.e. regular exercise once or twice per week for at least $30 \mathrm{~min}$ at each occasion, such as jogging, aerobics, weight training, soccer, etc.); and (4) regular exercise (i.e. regular exercise at least three times per week for at least $30 \mathrm{~min}$ at each occasion, such as jogging, aerobics, weight training, soccer etc.).

\section{Criterion measurement of $P A$}

PA was measured by the MTI Actigraph (Manufacturing Technology Inc., Fort Walton Beach, FL, USA; formerly known as the Computer Science and Application (CSA)) model 7164 activity monitor. This monitor is a uniaxial accelerometer measuring vertical accelerations. The monitor is small, lightweight $(\sim 45 \mathrm{~g})$ and provides detailed information about the intensity, frequency and duration of PA with sufficient storage capacity for monitoring PA minute-by-minute for 3 weeks. The technical specifications of the monitor are described elsewhere ${ }^{6}$. Briefly, accelerations ranging in magnitude from 0.05 to $2.0 \mathrm{~g}$ are measured by a piezoelectric sensor, sampled at $10 \mathrm{~Hz}$, and then summed over a selected time interval (epoch).

The activity monitor has been validated extensively during controlled laboratory settings in various groups of subjects, as well as during free-living conditions. Data from these studies suggest a relatively high degree of validity for quantifying the intensity of $\mathrm{PA}^{7-11}$. Additionally, average intensity (average counts $\min ^{-1}$ ) measured over several days has also been shown to be significantly associated with EE estimates measured by the doubly labelled water method under free-living conditions in children, adolescents, young athletes and adults ${ }^{12-15}$.

In the present study, the monitor was initialised as described by the manufacturer and a 1-min epoch was 
used. PA data were processed and analysed by a customwritten program (MAHUffe.exe, available from www.mrcepid.cam.ac.uk). Activity data were cleaned for periods when the monitor was not worn by excluding consecutive strings of zero-count epochs lasting 20 min or more. The main outcome variable from the activity monitor was the average intensity of PA (counts $\min ^{-1}$ ), calculated with equal weighting given to each day (regardless of registered time per day). We also calculated the amount of time spent in sedentary $\left(<100\right.$ counts $\left.\min ^{-1}\right)$, light (100-1952 counts min $\left.^{-1}\right)$, moderate (1952-5724 counts $\mathrm{min}^{-1}$ ) and vigorous (>5724 counts $\mathrm{min}^{-1}$ ) intensity of $\mathrm{PA}^{12}$. The threshold for time spent in sedentary activity is an arbitrary cut-off previously used in the global reliability and validity study of IPAQ ${ }^{4}$ and in other studies using accelerometry ${ }^{16}$. The subjects were asked to wear the accelerometer in a supplied elastic waist belt during waking hours except during water activities, over a 7-day period. According to the criterion for registered time, all subjects except one (not included in the analyses) recorded at least 5 days with more than 600 min of valid movement registration. The monitors were worn for an average of $762 \pm 84 \mathrm{~min}$ each day.

\section{Statistical analysis}

Values in tables are presented as mean \pm SD, unless otherwise stated. Self-reported PA variables, apart from time spent sitting, were skewed and were therefore logtransformed before analyses. Differences between genders were analysed by analysis of variance. Associations between self-reported and objectively measured PA were assessed using Pearson correlation. Multiple linear regression analyses were performed to assess the association between self-reported total amount of PA (MET-minday ${ }^{-1}$ ) and the total amount of objectively measured PA (total counts $\min ^{-1} \mathrm{day}^{-1}$ ), adjusting for gender, age, education and BMI. The interaction terms (age $\times$ counts, sex $\times$ counts, BMI $\times$ counts, education $\times$ counts) were included in preliminary models to test whether these variables modified the relationship between objectively measured and self-reported PA. No significant interactions were observed, so non-stratified models are presented here. Agreement between selfreported time spent in MVPA and measured time spent at the same intensity level by accelerometry was assessed with a modified Bland-Altman technique ${ }^{17}$. This analysis was used for assessing the absolute validity of the questionnaire. We plotted the difference between the criterion-measured (by accelerometry) time spent in MVPA and self-reported MVPA against the criterion. This modification of the Bland-Altman technique was used as the accelerometry-measured activity serves as the criterion in this comparison. In addition, concordance between the number of individuals meeting or not meeting the ACSM/CDC activity guidelines ${ }^{5}$, as determined by the two different methods, was assessed with the chi-square test. Sensitivity (ability of the IPAQ to identify not sufficiently active individuals) and specificity (ability of the IPAQ to identify sufficiently active individuals) for this measure were used to assess this other aspect of validity.

Furthermore, quartiles of self-reported total amount of activity (MET-min day ${ }^{-1}$ ) were compared with quartiles of accelerometer output using analysis of covariance after adjustment for gender, age, education and BMI. A similar analysis was performed to examine differences in MTI output between activity groups defined by the categorical SCB questions regarding leisure-time PA during the last 12 months and work-related activity. Two subjects reported no leisure-time PA, and were therefore combined with those who reported sporadic moderate leisure-time activity. Data were analysed by SPSS version 11 for Windows (SPSS Inc., Chicago, IL, USA) and the level of significance was set at $P<0.05$.

\section{Results}

The physical characteristics of the participants are displayed in Table 1. Also shown in Table 1 are the results obtained by accelerometry and self-reported PA from the IPAQ. Approximately 65\% of participants had a college degree or equivalent and less than $7 \%$ had no more than 9 years of compulsory education. There were no significant sex differences for objectively measured or self-reported derived PA variables. Overall, $47 \%$ of female and $40 \%$ of male participants reported no participation in vigorous-intensity PA. In addition, 35\% of females and 27\% of males reported no participation in walking during the previous week. In total, $15 \%$ of participants did not achieve the recommended amount of PA, as they reported MVPA and walking for less than $30 \mathrm{~min} \mathrm{day}^{-1}$ or less than 600 MET-min week $^{-1}$. When excluding walking, 30\% of participants were regarded as insufficiently active.

According to accelerometry data, males and females spent the majority of the day in sedentary behaviour. Roughly $54 \%$ of the registered time was spent at an intensity level $<100$ counts $\min ^{-1}$. No more than $6 \%$ of registered time was spent in moderate-intensity activity, or higher, without any sex difference. Thirty per cent of participants did not accumulate at least $30 \mathrm{minday}^{-1}$ at this intensity level.

The sensitivity of IPAQ to capture insufficiently active individuals was $45 \%$, whereas $77 \%$ (specificity) of those meeting the ACSM/CDC guidelines as determined by the activity monitor were captured by the questionnaire $\left(\chi^{2}=0.004\right)$. The positive and negative predictive values of the IPAQ were $45 \%$ and $77 \%$, respectively (Table 2).

In Table 3, associations between variables of selfreported PA and objectively measured PA are shown. Selfreported time spent sitting was significantly and positively correlated with time spent in sedentary behaviour by 
Table 1 Physical characteristics, objective physical activity (PA) measures and self-reported PA variables $(n=185)$

\begin{tabular}{|c|c|c|}
\hline & Males $(n=87)$ & Females $(n=98)$ \\
\hline Age (years) & $42.0 \pm 13$ & $41.6 \pm 13$ \\
\hline Weight $(\mathrm{kg})$ & $83.6 \pm 12.1$ & $64.8 \pm 10.3^{*}$ \\
\hline Height $(\mathrm{m})$ & $1.83 \pm 0.06$ & $1.67 \pm 0.06^{*}$ \\
\hline Body mass index $\left(\mathrm{kg} \mathrm{m}^{-2}\right)$ & $25.0 \pm 3.4$ & $23.2 \pm 3.3^{*}$ \\
\hline \multicolumn{3}{|l|}{ Objective PA measurements } \\
\hline Registered time $\left(\min\right.$ day $\left.^{-1}\right) \dagger$ & $766 \pm 82$ & $759 \pm 86$ \\
\hline Sedentary $\left(\min\right.$ day $\left.^{-1}\right) \dagger$ & $427 \pm 74$ & $394 \pm 69$ \\
\hline Light intensity $\left(\min\right.$ ay $\left.^{-1}\right) \dagger$ & $293 \pm 69$ & $320 \pm 70$ \\
\hline Moderate intensity $\left(\min ^{2} \mathrm{y}^{-1}\right) \dagger$ & $38 \pm 21$ & $40 \pm 20$ \\
\hline Vigorous intensity (min day $\left.{ }^{-1}\right) \dagger$ & $6.9 \pm 9.7$ & $5.2 \pm 9.1$ \\
\hline Total activity (counts min day ${ }^{-1}$ ) & $443 \pm 153$ & $473 \pm 151$ \\
\hline \multicolumn{3}{|l|}{ Self-reported variables } \\
\hline Sitting $\left(\mathrm{h} \mathrm{day}^{-1}\right) \ddagger$ & $6.9(4.6-8.6)$ & $6.9(5.2-8.9)$ \\
\hline Walking $\left(\right.$ min day $\left.^{-1}\right) \ddagger$ & $12.1(0-28.6)$ & $8.6(0-30.5)$ \\
\hline Moderate $\left(\min\right.$ day $\left.^{-1}\right) \ddagger$ & $12.1(0-31.2)$ & $20.4(4.5-34.3)$ \\
\hline Vigorous (min day $\left.{ }^{-1}\right) \ddagger$ & $8.6(0-25.7)$ & $3.6(0-17.1)$ \\
\hline Time in activity $\left(\min\right.$ day $\left.^{-1}\right) \ddagger$ & $46.4(30.0-82.1)$ & $51.4(28.6-85.7)$ \\
\hline Total activity $\left(\right.$ MET-min day $\left.{ }^{-1}\right) \ddagger$ & $211(120-419)$ & $228(126-397)$ \\
\hline
\end{tabular}

*, $P<0.0001$.

† Assessed by the MTI Actigraph (Manufacturing Technology Inc., Fort Walton Beach, FL, USA) activity monitor; values are mean \pm standard deviation.

$\ddagger$ Self-reported PA from the International Physical Activity Questionnaire; values are median (interquartile range).

Table 2 Number (\%) of subjects classified as being sufficiently active according to physical activity (PA) guidelines by self-report and by accelerometry $(n=185)$

\begin{tabular}{llcc}
\hline & \multicolumn{3}{c}{ Meeting } \\
\cline { 2 - 4 } Meeting & \multicolumn{3}{c}{ PA guideline, self-report } \\
\cline { 2 - 4 } PA guideline, accelerometry & \multicolumn{1}{c}{ No } & Yes & Total \\
\hline No & $25(45)$ & $30(23)$ & $55(30)$ \\
Yes & $30(55)$ & $100(77)$ & $130(70)$ \\
Total & $55(100)$ & $130(100)$ & $185(100)$ \\
\hline
\end{tabular}

Chi-square test for proportions $(P<0.001)$. accelerometry (i.e. $<100$ counts $\min ^{-1}$ ), and significantly and inversely correlated with activity time and the average intensity (counts $\mathrm{min}^{-1}$ ) from accelerometry. Similarly, self-reported activity time, time in MVPA and total activity $\left(\right.$ MET-min day $^{-1}$ ) were each significantly and inversely correlated with accelerometry-measured time spent in sedentary behaviour, and significantly and positively correlated with accelerometry-measured time spent in activity, time spent in MVPA and average PA intensity (counts $\min ^{-1}$ ), respectively.

Next we examined whether gender, age, education and BMI affected the association between the total amount of objectively measured PA (average counts $\min ^{-1}$ ) and self-reported PA (MET-minday ${ }^{-1}$ ). Total amount of

Table 3 Correlations (Pearson) between questionnaire-derived variables and objective measures of physical activity. Questionnaire-derived variables are log-transformed except for time spent sitting $(n=185)$

\begin{tabular}{|c|c|c|c|c|}
\hline \multirow[b]{2}{*}{ Objective variables } & \multicolumn{4}{|c|}{ Questionnaire-derived variables } \\
\hline & Sitting† & $\begin{array}{c}\text { Activity } \\
\text { timef }\end{array}$ & MVPA§ & Total activity \\
\hline Sedentary $\left(\min\right.$ day $\left.^{-1}\right) \|$ & $0.16^{*}$ & $-0.19^{\star *}$ & $-0.22^{\star *}$ & $-0.19^{* *}$ \\
\hline Light + moderate + vigorous $\left(\min\right.$ day $\left.^{-1}\right) \dagger \dagger$ & $-0.35^{\star * *}$ & $0.17^{*}$ & $0.20^{*}$ & $0.18^{\star}$ \\
\hline Moderate + vigorous $\left(\min\right.$ day $\left.^{-1}\right) \neq \ddagger$ & -0.07 & $0.21^{* *}$ & $0.17^{\star}$ & $0.30^{\star * \star}$ \\
\hline Total activity (average counts $\min ^{-1}$ ) & $-0.16^{\star}$ & $0.21^{* *}$ & $0.22^{* *}$ & $0.34^{\star \star \star}$ \\
\hline
\end{tabular}

${ }^{\star}, P<0.05 ;{ }^{* \star}, P<0.01 ;{ }^{* \star \star}, P<0.001$.

† Self-reported time sitting.

$\ddagger$ Self-reported time in activity (walking + moderate + vigorous).

$\S$ Self-reported time in moderate and vigorous physical activity.

I Total activity (MET-min day ${ }^{-1}$ ).

II Sedentary; $<100$ counts min $^{-1}$

†† Activity; $\geq 100$ counts min $^{-1}$

$¥$ Moderate and vigorous activity; $\geq 1952$ counts $\mathrm{min}^{-1}$. 
self-reported PA was the only variable which contributed significantly to the explained variation in objectively measured PA (adjusted $R^{2}=0.14 ; P<0.0001$ ).

Figure 1a illustrates the difference between objectively measured time spent in MVPA and self-reported PA plotted against the accelerometry estimates of MVPA (modified Bland-Altman plot). The mean difference between methods was $3.4 \mathrm{minday}^{-1}$ (not significant); however,
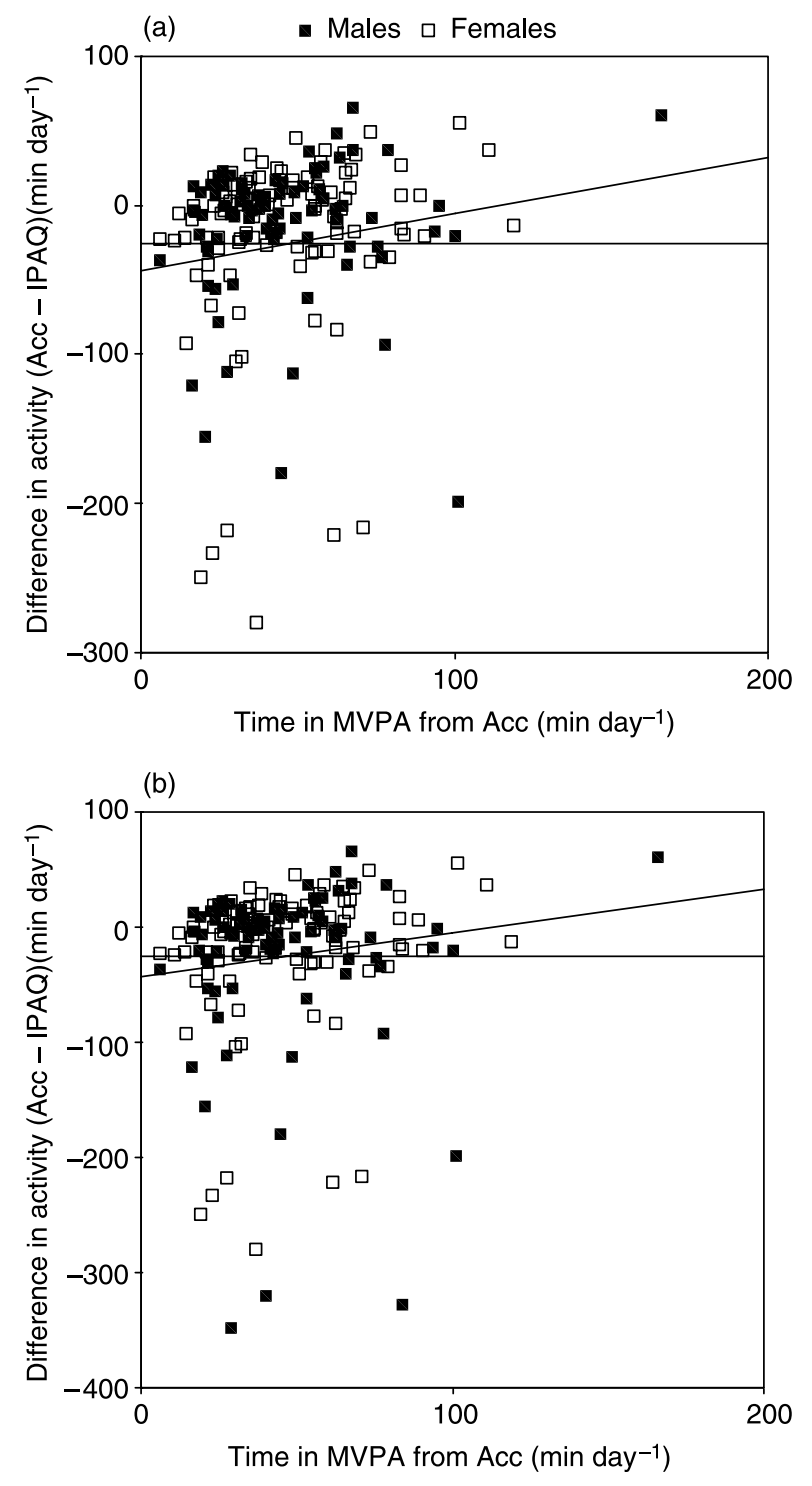

Fig. 1 (a) The difference between objectively measured time (determined by accelerometry, Acc) and self-reported time (determined by the International Physical Activity Questionnaire, IPAQ) spent in moderate and vigorous physical activity (MVPA) $\left(\right.$ min day $\left.^{-1}\right)$ plotted against time spent in MVPA from Acc $\left(\min\right.$ day $\left.^{-1}\right)$. Mean difference: 3.4 min day $^{-1}$; limits of agreement: -119 to 122 min day $^{-1}(n=185)$. (b) The difference between objectively measured time (AcC) and selfreported time (IPAQ) spent in MVPA and walking (min day ${ }^{-1}$ ) plotted against time spent in MVPA from Acc (min day ${ }^{-1}$ ). Mean difference: -25.9 min $^{-1}{ }^{-1}$; limits of agreement: -172 to 120 min day $^{-1}(n=185)$. The difference between methods was not significantly correlated to time spent at MVPA from Acc (both $r=0.11, P=0.12$ )

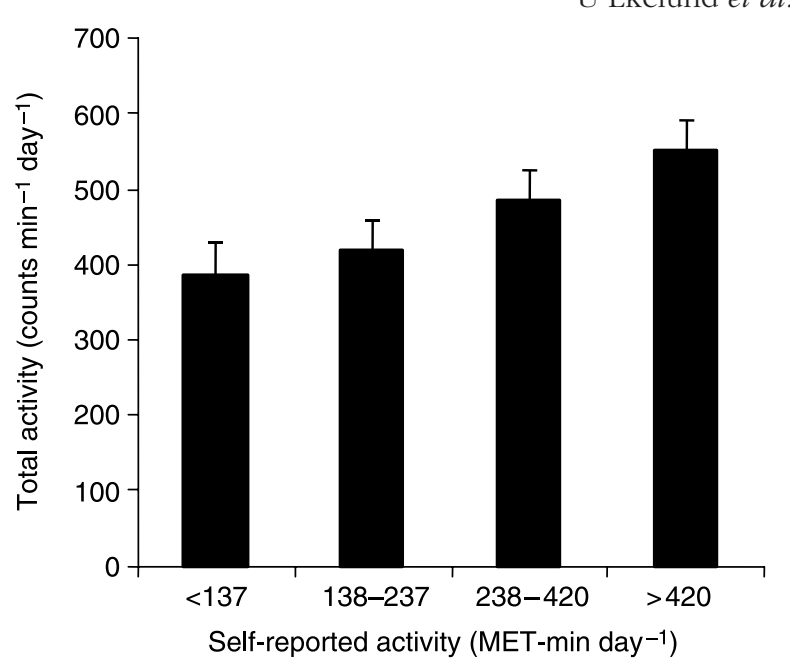

Fig. 2 Mean total amount of physical activity assessed by the activity monitor stratified by quartiles of self-reported physical activity (MET-minday ${ }^{-1}$ ); bars show 95\% confidence intervals. Data are adjusted for age, gender, education and body mass index $(P$ for trend $<0.001 ; n=185)$

the $95 \%$ limits of agreement were wide $(-119$ to 122 min day $^{-1}$ ). In Fig. 1b, the difference between objectively measured time and self-reported time in MVPA and walking is plotted against the accelerometry estimates of MVPA. The mean difference was -25.9 min day $^{-1}(P<0.001)$ and the $95 \%$ limits of agreement were wide ( -172 to 120 min day $^{-1}$ ).

Figure 2 shows the average intensity of PA (counts $\min ^{-1}$ ) by quartiles of self-reported total amount of PA (MET-min day ${ }^{-1}$ ). Post hoc analyses (Tukey) revealed significant differences between quartiles 1 and $3(P=0.006)$, between quartiles 1 and $4(P<0.001)$ and between quartiles 2 and $4(P<0.001)$, after adjustment for gender, age and BMI.

Figure 3 displays the sample mean of the average intensity of PA (counts $\mathrm{min}^{-1}$ ) by self-reported leisuretime PA during the last 12 months according to the question used in the ULF surveys. Data are adjusted for age, gender and BMI. Post hoc analyses (Tukey) revealed that those who reported no or sporadic participation in leisure-time PA were significantly less active than those who reported participation in moderate PA once or twice per week $(P=0.034)$, and compared with those who reported participation in regular exercise and sports more than twice per week $(P<0.0001)$. We did not observe any difference in objectively measured PA between groups of self-reported PA at work during the last 12 months.

\section{Discussion}

In the present paper we report the criterion-related and absolute validity for the last 7-day, short-form IPAQ for use in Swedish adults. Our results demonstrate moderate criterion validity $(r=0.16-0.35)$ for most of the activity indicators derived from the IPAQ. To our knowledge, this 


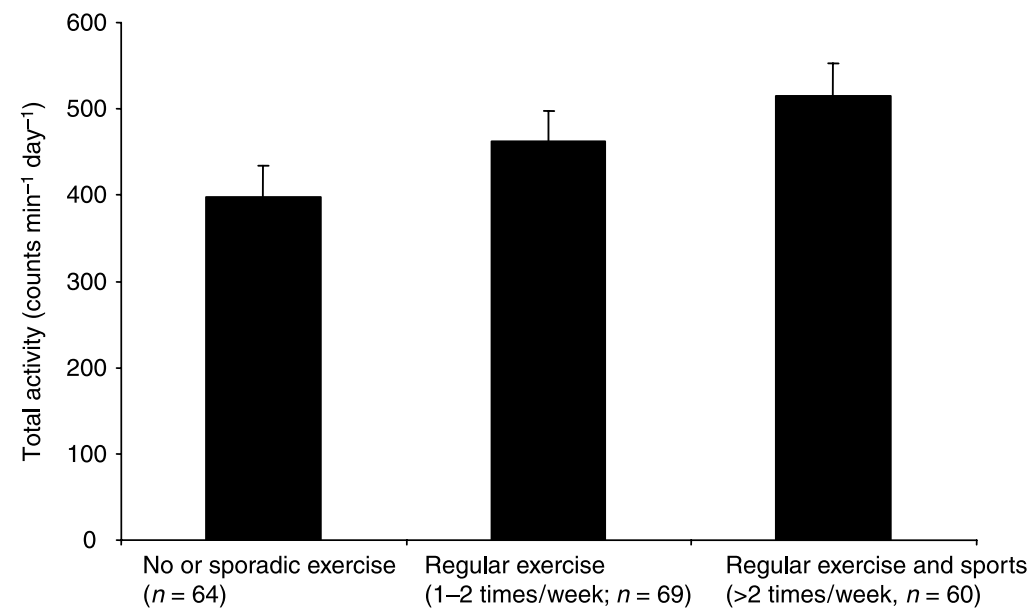

Fig. 3 Mean total amount of physical activity objectively measured by accelerometry and self-reported leisure-time exercise during the last 12 months; bars show 95\% confidence intervals. Data are adjusted for age, gender, education and body mass index $(P$ for trend $<0.001 ; n=185)$

study is the first to evaluate the absolute time spent in MVPA from the IPAQ questionnaire. We observed no significant difference (mean difference: 3.4 min day $^{-1}$ ) between self-reported and objectively measured MVPA. However, when self-reported walking was included in this analysis, a highly significant mean difference between methods was observed. The absolute validity of the IPAQ is therefore probably low. Furthermore, the wide limits of agreement indicate that the IPAQ is unable to assess MVPA on an individual basis (Fig. 1). The IPAQ correctly classified $77 \%$ of respondents as sufficiently physically active, whereas the sensitivity to identify individuals in need of increasing their physical activity was poor (45\%).

Assessment of the validity of questionnaires is difficult, but the study design adopted here allowed for direct measurement of PA by the activity monitor during the time period referred to by the questionnaire. We have therefore no reason to believe our respondents did not refer to the same days when answering the questionnaire as have been measured by the activity monitor. This is of particular importance when addressing the absolute validity of the instrument. The majority of subjects were selected randomly from different workplaces in the fourth largest town in Sweden (Uppsala, approximately 180000 inhabitants). The recruitment from different workplaces allowed us to obtain a sample with reasonable heterogeneity in age and education level. None the less, mean BMI in women was significantly lower (23.2 vs. $24.4 \mathrm{~kg} \mathrm{~m}^{-2}$ ) than the Swedish reference values ${ }^{18}$, whereas the mean BMI in men was similar ( 25.0 vs. $25.4 \mathrm{~kg} \mathrm{~m}^{-2}$ ). Since our study sample was more educated and borderline leaner than the general Swedish population, this may limit the generalisability of our results. However, the significant association between accelerometer-measured PA and selfreported PA was not affected by age, sex, education and BMI within the sample, suggesting this limitation may be minor. With few exceptions ${ }^{19}$, previous studies evaluating the validity of PA questionnaires often include selected samples of volunteers ${ }^{4,20-22}$.

The comparison between accelerometry and selfreported PA needs to be interpreted with caution. When deriving the time estimate from the accelerometry data, i.e. time spent in MVPA, all minutes spent above the predetermined threshold for MVPA (i.e. 1952 counts $\min ^{-1}$ ) were included, whereas the questionnaire prompted for 10-min blocks of activity. In fact, if only 10min blocks of MVPA from the activity monitor are compared with the self-reported estimate, the difference between methods is even greater (data not shown). Our participants spent on average slightly more than one continuous 10-min block in MVPA per day. The time spent at different intensity levels assessed by accelerometry depends on the intensity thresholds applied and in turn on the calibration activities performed to establish the relationship between activity counts and energy expenditure. Thus, the observed agreement between self-reported and objectively measured MVPA needs to be interpreted also bearing this in mind. Furthermore, accelerometry has limitations as it does not accurately record body movement during specific activities such as bicycling and climbing stairs, and cannot be used during water activities. Finally, we reported all our comparisons between selfreported and objectively measured PA with and without self-reported walking, since it may be more difficult to accurately quantify the amount of time spent walking.

Our results indicated that more than $75 \%$ of those who reported sufficient physical activity according to the ACSM/CDC guidelines by IPAQ also were active for more than 30 min day $^{-1}$ according to the accelerometer. In contrast, no more than $45 \%$ of those who did not meet the guidelines were correctly classified as insufficiently active by IPAQ. In other words, the IPAQ provides a reasonably specific measure of PA whereas its sensitivity to correctly classify inactive people is limited. 
We observed significant associations for most of the derived self-report measures in comparison with objectively measured PA. Correlation coefficients ranged from 0.16 to 0.35 , indicating moderate criterion validity of the IPAQ instrument. These correlation coefficients are similar to those obtained in the 12-country reliability and validity study of IPAQ ${ }^{4}$. However, in that study no significant associations were observed between self-reported PA and any of the objectively derived measures of activity for the Swedish sample ${ }^{4}$. This might have been due to the relatively small and selected study sample in the Swedish part of that study, being only a quarter the size of our sample in the present study. It is therefore reassuring that the results from the bigger study indicate that the last 7day, short-form IPAQ appears valid for use in a randomly selected sample of Swedish adults. Furthermore, we did not observe any influence of sex, age, education or BMI on the association between self-reported total amount of PA and objectively measured activity. This is in contrast to a previous study indicating a higher validity for self-reported PA in Swedish men with lower BMI in comparison to those with higher BMI $\left(>26 \mathrm{~kg} \mathrm{~m}^{-2}\right)^{23}$. However, a different criterion instrument (activity record) was used in that study which may have correlated error and thus explain the different findings. Our results suggest that the validity of PA collected retrospectively by self-report is not influenced by sex, age, education and BMI.

We also evaluated the validity of two questions (ULF; $\mathrm{SCB})$ regarding leisure-time $\mathrm{PA}$ and work-related activity during the last 12 months. The leisure-time PA question asked the respondent to categorise themselves into one of four categories of leisure-time PA. Only two individuals regarded themselves as being 'sedentary' during leisure time and were therefore combined with those who regarded themselves as 'somewhat active'. We observed significant differences in activity counts between groups, indicating that this question may be valid for categorising people into different categories of activity (Fig. 3). In contrast, we did not observe any differences in objectively measured PA between groups according to work-related activity. This may partially be explained by the fact that few $(n=9)$ participants were involved in heavy manual labour in our study sample. However, it may also indicate that leisure-time PA is more important than work-related activity when contributing to the total amount of PA in adults. Future studies utilising accelerometry may address this issue by an hour-by-hour comparison for activity counts during working hours compared with leisure time.

In conclusion, our results indicate moderate criterion validity of the short, last 7-days form of the IPAQ for use in Swedish adults, indicating its usefulness for ranking and categorising respondents into activity categories. However, the IPAQ instrument significantly overestimated selfreported time spent in PA. In terms of correctly classifying individuals into whether or not they meet current PA guidelines, the specificity to identify sufficiently active individuals was acceptable whereas the sensitivity to identify insufficiently active was poor.

\section{Acknowledgements}

This study was funded by the Swedish National Institute of Public Health and the Swedish National Center for Research in Sports. The authors are grateful to all volunteers who participated in the study.

\section{References}

1 Caspersen CJ, Powell K, Christenson G. Physical activity, exercise, and physical fitness: definitions and distinctions for health-related research. Public Health Reports 1985; 100. 126-31.

2 Kriska AM, Caspersen CJ. A collection of physical activity questionnaires for health-related research. Medicine and Science in Sports and Exercise 1997; 29: S5-9.

3 Wareham NJ, Rennie K. The assessment of physical activity in individuals and populations: why try to be more precise about how physical activity is assessed? International Journal of Obesity and Related Metabolic Disorders 1998; 22: $\mathrm{S} 30-8$.

4 Craig CL, Marshall AL, Sjöström M, Bauman AE, Booth ML, Ainsworth BE, et al. International physical activity questionnaire: 12 -country reliability and validity. Medicine and Science in Sports and Exercise 2003; 35: 1381-95.

5 Pate RR, Pratt M, Blair S, Haskell WL, Macera CA, Bouchard C, et al. Physical activity and public health. A recommendation from the Centers for Disease Control and Prevention and the American College of Sports Medicine. Journal of the American Medical Association 1995; 273: 402-7.

6 Tryon W, Williams R. Fully proportional actigraphy: a new instrument. Behavior Research Methods, Instruments, \& Computers 1996; 28: 392-403.

7 Brage S, Wedderkopp N, Franks PW, Andersen LB, Froberg K. Reexamination of validity and reliability of the CSA monitor in walking and running. Medicine and Science in Sports and Exercise 2003; 35: 1447-54.

8 Freedson P, Melanson E, Sirard J. Calibration of the Computer Science and Applications, Inc. accelerometer. Medicine and Science in Sports and Exercise 1998; 30: 777-81.

9 Hendelman D, Miller K, Baggett C, Debold E, Freedson P. Validity of accelerometry for the assessment of moderate intensity physical activity in the field. Medicine and Science in Sports and Exercise 2000; 32: S442-9.

10 Trost S, Ward D, Moorehead S, Watson P, Riner W, Burke J. Validity of the Computer Science and Applications (CSA) activity monitor in children. Medicine and Science in Sports and Exercise 1998; 30: 629-33.

11 Ekelund U, Tingström P, Kamwendo K, Krantz M, Nylander E, Sjöström $\mathrm{M}$, et al. The validity of the Computer Science and Applications activity monitor for use in coronary artery disease patients during level walking. Clinical Physiology and Functional Imaging 2002; 22: 248-53.

12 Ekelund U, Sjöström M, Yngve A, Poortvliet E, Nilsson A, Froberg K, et al. Physical activity assessed by activity monitor and doubly labeled water in children. Medicine and Science in Sports and Exercise 2001; 33: 275-81.

13 Ekelund U, Aman J, Westerterp K. Is the ArteACC index a valid indicator of free-living physical activity in adolescents? Obesity Research 2003; 11: 793-801.

14 Leenders NY, Sherman WM, Nagaraja HN, Kien CL. Evaluation of methods to assess physical activity in freeliving conditions. Medicine and Science in Sports and Exercise 2001; 33: 1233-40. 
15 Ekelund U, Yngve A, Sjöström M, Westerterp K. Field evaluation of the Computer Science and Application's Inc. activity monitor during running and skating training in adolescent athletes. International Journal of Sports Medicine 2000; 21: 586-92.

16 Ekelund U, Aman J, Yngve A, Renman C, Westerterp K, Sjöstrom M. Physical activity but not energy expenditure is reduced in obese adolescents: a case-control study. American Journal of Clinical Nutrition 2002; 76: 935-41.

17 Bland JM, Altman DG. Statistical methods for assessing agreement between two methods of clinical measurement. Lancet 1986; 1(8476): 307-10.

18 Sundquist K, Qvist J, Johansson SE, Sundquist J. Increasing trends of obesity in Sweden between 1996/97 and 2000/01. International Journal of Obesity and Related Metabolic Disorders 2004; 28: 254-61.

19 Wareham NJ, Jakes RW, Rennie KL, Mitchell J, Hennings S, Day NE. Validity and repeatability of the EPIC-Norfolk
Physical Activity Questionnaire. International Journal of Epidemiology 2002; 31: 168-74.

20 Jacobs DR Jr, Ainsworth BE, Hartman TJ, Leon AS. A simultaneous evaluation of 10 commonly used physical activity questionnaires. Medicine and Science in Sports and Exercise 1993; 25: 81-91.

21 Chasan-Taber L, Erickson JB, Nasca PC, Chasan-Taber S, Freedson PS. Validity and reproducibility of a physical activity questionnaire in women. Medicine and Science in Sports and Exercise 2002; 34: 987-92.

22 Aadahl M, Jorgensen T. Validation of a new self-report instrument for measuring physical activity. Medicine and Science in Sports and Exercise 2003; 35: 1196-202.

23 Norman A, Bellocco R, Bergström A, Wolk A. Validity and reproducibility of self-reported total physical activity differences by relative weight. International Journal of Obesity and Related Metabolic Disorders 2001; 25: 682-8. 